\title{
Using Computer Algebra Systems together with lavaan to generate symbolic model-implied covariance matrices in SEM
}

\author{
Henrik Kenneth Andersen \\ 25 September 2019
}

This article is meant as (brief, introductory) guide to using computer algebra systems to work out analytical model-implied covariance matrices for structural equation models. To my knowledge there is no pre-packaged software available for this task. The article is meant primarily for those with experience with structural equation modeling as well as lavaan and ideally $\mathrm{R}$ in general.

Any questions or comments can be sent to henrik.andersen@soziologie.tu-chemnitz.de.

\section{Introduction}

A careful inspection of the model-implied covariance matrix should be considered an essential step in any SEM-based regression model. Software default settings, cluttered or inaccurate path diagrams as well as an over reliance on modification indices often hide important aspects of SEM-based regression models. In complex models, researchers may not even be aware of the underlying algebra that is responsible for the reported parameter estimates.

This brief article demonstrates the use of Computer Algebra Systems (CAS) such as Ryacas (Andersen et al. 2019), an R interface with the software Yacas ("Yet Another Computer Algebra System"), along with the popular Structural Equation Modeling (SEM) package lavaan (Rosseel 2012) to generate symbolic representations of model-implied covariance matrices.

The model-implied covariance matrix can be derived using traditional methods such as the tracing rule, or with covariance algebra. However, these methods are a) tedious, b) prone to errors, c) must be done by hand and entered into the software of choice later. The method outlined here involves entering the model matrices into Ryacas and letting the software come up with the entire symbolic covariance matrix in one go. It saves time, is less error prone and can reveal details that may not be obvious (especially with more complex models or ones with latent variables, e.g. fixed-effects regression in SEM).

The article is outlined as follows: first I simulate a simple mediation model with manifest indicators to be used as the dataset in the later demonstration. Then I outline the model matrices used in popular SEM software like lavaan and Mplus and derive the equation for the model-implied covariance matrix. I then run the model as outlined in the simulation in lavaan and show how to double-check the model matrices. Before demonstrating the CAS-based approach, I give an example of generating the model-implied covariance between just two model variables algebraically. After doing so, I then introduce the CAS-based approach. This involves introducing the model elements as symbols into Ryacas and entering in the model matrices. Then I generate the entire symbolic model-implied covariance matrix and show that the procedure does in fact give the proper results. Lastly, I show just one example of how a close inspection of the model-implied covariance matrix can help researchers gain a better understanding of their SEM models. This applies regardless of the method of generating the model-implied covariance matrix. The CAS-based approach can be seen as another available tool towards this goal.

\section{Set-up}

The only packages needed are lavaan and Ryacas. 


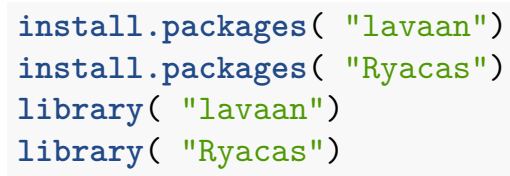

Let us simulate a simple model using tools available in base R (R Core Team 2017) to use as our dataset. That way, the 'true' parameter values are known so that it will be possible to later double-check the work.

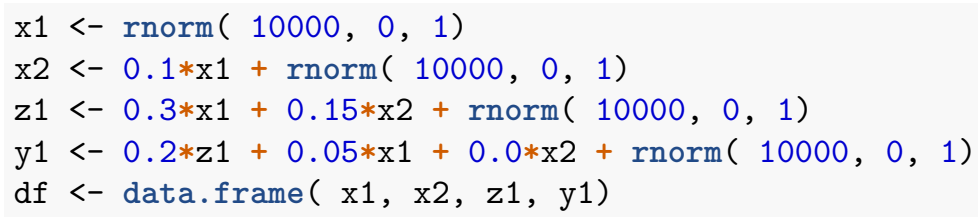

I chose a sample size of $n=10,000$ in order to minimize but not eliminate sampling error, and each of the exogenous (independent) variables in the model $\left(x_{1}, \delta_{2}, v_{1}\right.$ and $\left.\varepsilon_{1}\right)$ are $N \sim(0,1)$.

The empirical covariance matrix can be viewed with

$\operatorname{cov}(\mathrm{df})$

$\begin{array}{lrrrrr}\text { \#\# } & \mathrm{x} 1 & \mathrm{x} 2 & \mathrm{z} 1 & \mathrm{y} 1 \\ \text { \#\# } & \mathrm{x} 1 & 0.98880443 & 0.09639345 & 0.3002384 & 0.10500371 \\ \text { \#\# } \mathrm{x} 2 & 0.09639345 & 0.99990831 & 0.1850999 & 0.03891528 \\ \text { \#\# } & \mathrm{z} 1 & 0.30023840 & 0.18509987 & 1.1147841 & 0.24146647 \\ \text { \#\# } & \mathrm{y} 1 & 0.10500371 & 0.03891528 & 0.2414665 & 1.07471688\end{array}$

We can write equations for each of the model variables

$$
\begin{aligned}
x_{1} & =\delta_{1} \\
x_{2} & =\varphi_{1} x_{1}+\delta_{2} \\
z_{1} & =\gamma_{1} x_{1}+\gamma_{2} x_{2}+v_{1} \\
y_{1} & =\beta_{1} x_{1}+\beta_{2} z_{1}+\varepsilon_{1}
\end{aligned}
$$

where, from the code above, we know $\varphi_{1}=0.1, \gamma_{1}=0.3, \gamma_{2}=0.15, \beta_{1}=0.05, \beta_{2}=0.2$. Notice we have also written an equation for the only observed exogenous variable, $x_{1}=\delta_{1}$, which basically states that the entire variance is due to influences not contained in the model. This will be important to note below when we specify the model matrices. The path diagram of our model is shown in Figure 1.

\section{Model matrices}

The SEM-matrix notation I use most closely mimics the one used in popular SEM software like lavaan and Mplus. It has been outlined by (Bollen 1989; Graff 1979) The basic model is

$$
\begin{aligned}
& \boldsymbol{\eta}=\boldsymbol{B} \boldsymbol{\eta}+\boldsymbol{\zeta} \\
& \boldsymbol{y}=\boldsymbol{\Lambda} \boldsymbol{\eta} .
\end{aligned}
$$

If $m$ stands for the number of independent latent variables, $n$ is the number of dependent latent variables, $p$ is the number of independent observed (manifest) variables and $q$ is the number of dependent observed variables, then the $(m+n+p+q \times 1)$ vector $\boldsymbol{\eta}$ holds the observed and latent variables, $\boldsymbol{\zeta}$ is also $(m+n+p+q \times 1)$ 


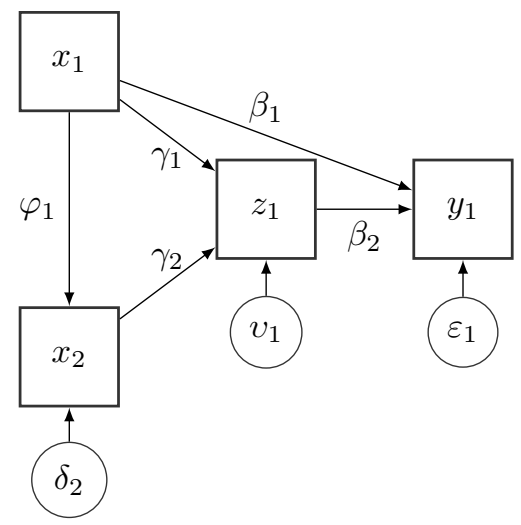

Figure 1: Path diagram

and holds any latent variables and the errors of the manifest variables and $\boldsymbol{y}$ is $(p+q \times 1)$ and holds only the observed variables. The matrix $\boldsymbol{\Lambda}$ is $(p+q \times m+n+p+q)$ and has only ones and zeros as elements and selects the observed variables from $\boldsymbol{\eta}$. The $\boldsymbol{\Psi}$ matrix (introduced below) is $(m+n+p+q \times m+n+p+q)$ and specifies the (co-)variances of the variables in $\zeta$. Finally, the $\mathbf{B}$ matrix is also $(m+n+p+q \times m+n+p+q)$ and holds the model coefficients.

For this example model, we have: $\boldsymbol{\eta}^{\prime}=\left(x_{1}, x_{2}, z_{1}, y_{1}\right), \boldsymbol{\zeta}^{\prime}=\left(\delta_{1} \delta_{2}, v_{1}, \varepsilon_{1}\right)$ and $\boldsymbol{y}^{\prime}=\left(x_{1}, x_{2}, z_{1}, y_{1}\right)$. Because there are no latent variables in this example, $\boldsymbol{\eta}$ is identical to $\boldsymbol{y}$ and the matrix $\boldsymbol{\Lambda}$ is an identity matrix

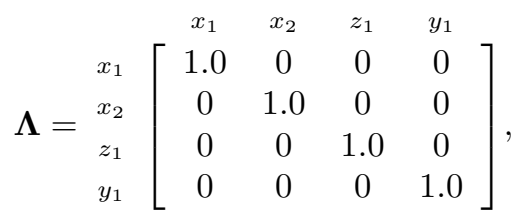

and $\boldsymbol{B}$ will be

$$
\boldsymbol{B}=\begin{gathered}
x_{1} \\
x_{2} \\
z_{1} \\
y_{1}
\end{gathered}\left[\begin{array}{cccc}
x_{1} & x_{2} & z_{1} & y_{1} \\
0 & 0 & 0 & 0 \\
\varphi_{1} & 0 & 0 & 0 \\
\gamma_{1} & \gamma_{2} & 0 & 0 \\
\beta_{1} & 0 & \beta_{2} & 0
\end{array}\right] .
$$

The $\boldsymbol{\Psi}$ matrix is important as it specifies the covariances of the independent variables (including the errors), essentially laying out all the model assumptions. Before showing the $\boldsymbol{\Psi}$ matrix we must first derive the the $\boldsymbol{\Sigma}(\boldsymbol{\theta})$ model-implied covariance matrix of the observed variables analytically. To do so, we first put $\boldsymbol{\eta}$ in reduced form

$$
\begin{aligned}
\boldsymbol{\eta} & =\mathbf{B} \boldsymbol{\eta}+\boldsymbol{\zeta} \\
& =(\boldsymbol{I}-\mathbf{B})^{-1} \boldsymbol{\zeta}
\end{aligned}
$$

and find the expected value of $\boldsymbol{y} \boldsymbol{y}^{\prime}$ 


$$
\begin{aligned}
\boldsymbol{y} \boldsymbol{y}^{\prime} & =(\boldsymbol{\Lambda} \boldsymbol{\eta})(\boldsymbol{\Lambda} \boldsymbol{\eta})^{\prime} \\
& =\left(\boldsymbol{\Lambda}(\boldsymbol{I}-\mathbf{B})^{-1} \boldsymbol{\zeta}\right)\left(\boldsymbol{\Lambda}(\boldsymbol{I}-\mathbf{B})^{-1} \boldsymbol{\zeta}\right)^{\prime} \\
& =\left(\boldsymbol{\Lambda}(\boldsymbol{I}-\mathbf{B})^{-1} \boldsymbol{\zeta}\right)\left(\boldsymbol{\zeta}^{\prime}(\boldsymbol{I}-\mathbf{B})^{-1 \prime} \boldsymbol{\Lambda}^{\prime}\right) \\
& =\boldsymbol{\Lambda}(\boldsymbol{I}-\mathbf{B})^{-1} \boldsymbol{\zeta} \boldsymbol{\zeta}^{\prime}(\boldsymbol{I}-\mathbf{B})^{-1 \prime} \boldsymbol{\Lambda}^{\prime} \\
E\left[\boldsymbol{y} \boldsymbol{y}^{\prime}\right] & =\boldsymbol{\Lambda}(\boldsymbol{I}-\mathbf{B})^{-1} E\left[\boldsymbol{\zeta} \boldsymbol{\zeta}^{\prime}\right](\boldsymbol{I}-\mathbf{B})^{-1 \prime} \boldsymbol{\Lambda}^{\prime} \\
\boldsymbol{\Sigma}(\boldsymbol{\theta}) & =\boldsymbol{\Lambda}(\boldsymbol{I}-\mathbf{B})^{-1} \boldsymbol{\Psi}(\boldsymbol{I}-\mathbf{B})^{-11} \boldsymbol{\Lambda}^{\prime} .
\end{aligned}
$$

It should be clear from this that the model is specified primarily according to the assumptions we place on $\boldsymbol{B}$ and $\boldsymbol{\Psi} .{ }^{1}$ In $\boldsymbol{B}$ we are deciding how the variables are related to one another and in $\boldsymbol{\Psi}$ we are (often implicitly) stating our further model assumptions. In this example, we are assuming that the model errors are uncorrelated with the exogenous variable $x_{1}$ and with each other (see the path diagram in Figure 1). Therefore, $\boldsymbol{\Psi}$ has the variances of the exogenous variables on the diagonal and zeros everywhere else

$$
\boldsymbol{\Psi}=\begin{aligned}
& x_{1} \\
& x_{2} \\
& z_{1} \\
& y_{1}
\end{aligned}\left[\begin{array}{cccc}
x_{1} & x_{2} & z_{1} & y_{1} \\
\sigma_{\delta_{1}}^{2} & 0 & 0 & 0 \\
0 & \sigma_{\delta_{2}}^{2} & 0 & 0 \\
0 & 0 & \sigma_{v_{1}}^{2} & 0 \\
0 & 0 & 0 & \sigma_{\varepsilon_{1}}^{2}
\end{array}\right],
$$

(keeping in mind that $x_{1}$ is exogenous, so $x_{1}=\delta_{1}$ ).

\section{Running the model in lavaan}

Having now reached our model specification, we can compare the matrices outlined above to the ones that lavaan used to come to the estimates. First though, the model is run to check the estimates. I use labels, e.g. $\mathrm{x} 2 \sim \mathrm{p} 1 * \mathrm{x} 1$, to help remind me later that the coefficient for the regression of $x_{2}$ on $x_{1}$ was called "phi one".

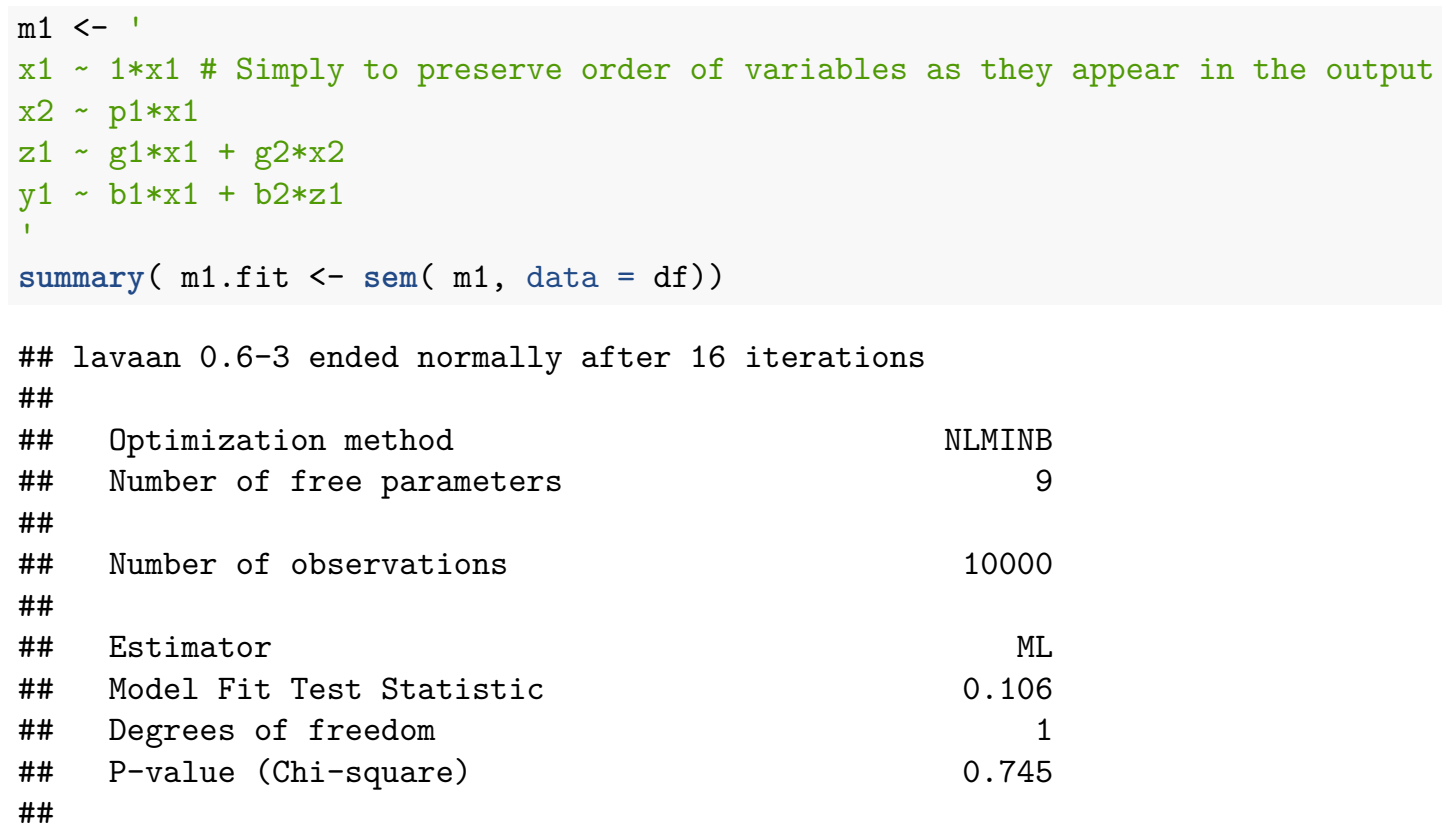

${ }^{1}$ The only choices we make regarding the vectors $\boldsymbol{\eta}, \boldsymbol{\zeta}$ and $\boldsymbol{y}$ are which variables to include in the model in the first place. 


\begin{tabular}{|c|c|c|c|c|c|c|}
\hline \#\# & Information & & & & & Expected \\
\hline \#\# & Information & satura & ated (h1) & model & & tructured \\
\hline \#\# & Standard Err & rors & & & & Standard \\
\hline \multicolumn{7}{|l|}{ \#\# } \\
\hline \multicolumn{7}{|c|}{ \#\# Regressiol } \\
\hline \#\# & & & Estimate & Std.Err & z-value & $P(>|z|)$ \\
\hline \multicolumn{7}{|c|}{$\mathrm{x} 1 \sim$} \\
\hline \#\# & $\mathrm{x} 1$ & & 1.000 & & & \\
\hline \multicolumn{7}{|c|}{$\mathrm{x} 2 \sim$} \\
\hline \#\# & $\mathrm{x} 1$ & (p1) & 0.097 & 0.010 & 9.740 & 0.000 \\
\hline \multicolumn{7}{|c|}{$\mathrm{z} 1 \sim$} \\
\hline \#\# & $\mathrm{x} 1$ & (g1) & 0.288 & 0.010 & 28.546 & 0.000 \\
\hline \#\# & $\mathrm{x} 2$ & (g2) & 0.157 & 0.010 & 15.665 & 0.000 \\
\hline \multicolumn{7}{|c|}{$\mathrm{y} 1$ } \\
\hline \#\# & $\mathrm{x} 1$ & (b1) & 0.044 & 0.011 & 4.152 & 0.000 \\
\hline \#\# & z1 & (b2) & 0.205 & 0.010 & 20.504 & 0.000 \\
\hline \multicolumn{7}{|l|}{ \#\# } \\
\hline \multicolumn{7}{|c|}{ \#\# Variances: } \\
\hline \#\# & & & Estimate & Std.Err & z-value & $P(>|z|)$ \\
\hline \#\# &.$x 1$ & & 0.989 & 0.014 & 70.711 & 0.000 \\
\hline \#\# &.$x 2$ & & 0.990 & 0.014 & 70.711 & 0.000 \\
\hline \#\# &.$z 1$ & & 0.999 & 0.014 & 70.711 & 0.000 \\
\hline \#\# & $\cdot \mathrm{y}_{1}$ & & 1.021 & 0.014 & 70.711 & 0.000 \\
\hline
\end{tabular}

Because of the large sample size, the estimated parameters are very close to the ones we used to generate the data. This should come as no surprise because we simply copied the data-generating process into our code and let the software come up with the estimates. Now, we can inspect the model matrices using the inspect() function in lavaan, where 'est' returns a list of the model matrices

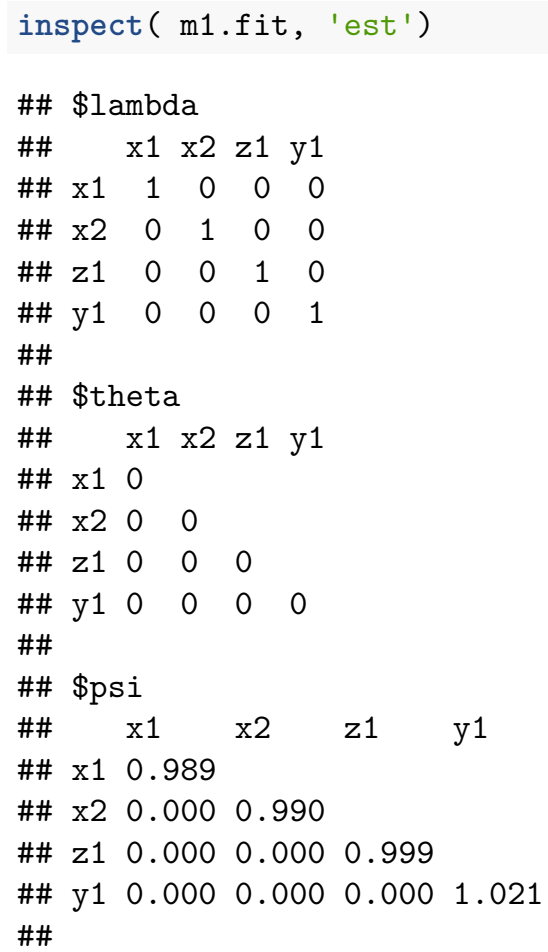




$\begin{array}{lrrrrr}\text { \#\# } & \text { \$beta } & & & & \\ \text { \#\# } & & \text { x1 } & \text { x2 } & \text { z1 } & \text { y1 } \\ \text { \#\# } & \text { x1 } & 1.000 & 0.000 & 0.000 & 0 \\ \text { \#\# } & \text { x2 } & 0.097 & 0.000 & 0.000 & 0 \\ \text { \#\# } & \text { z1 } & 0.288 & 0.157 & 0.000 & 0 \\ \text { \#\# } & \text { y1 } & 0.044 & 0.000 & 0.205 & 0\end{array}$

Here we see the matrices used to estimate the model are, in fact, the exact same ones we outlined above. The only difference is that here the estimated coefficients have been substituted in already.

\section{Model-implied covariance matrix}

\section{Typical approaches}

If we are interested in looking at how the parameter estimates were found, we need to look closer at the model-implied covariances.

It is of course possible to use the tracing rule (Kenny 1979) to come up with the underlying model-implied covariances but this is inefficient, tedious and it must be done by hand. Another option is to find the algebraic equation per covariance which is straightforward, but also tedious. For example, if we want to know how the effect $x_{1} \stackrel{\beta_{1}}{\longrightarrow} y_{1}$ was estimated, then we need to look at $\operatorname{Cov}\left(y_{1}, x_{1}\right)^{2}$

$$
\begin{aligned}
\operatorname{Cov}\left(y_{1}, x_{1}\right) & =E\left[y_{1} x_{1}\right] \\
& =E\left[\left(\beta_{1} x_{1}+\beta_{2} z_{1}+\varepsilon_{1}\right)\left(x_{1}\right)\right] \\
& =E\left[\beta_{1} x_{1}^{2}+\beta_{2} z_{1} x_{1}+\varepsilon_{1} x_{1}\right] \\
& =\beta_{1} \operatorname{Var}\left(x_{1}\right)+\beta_{2} E\left[z_{1} x_{1}\right] \\
& =\beta_{1} \operatorname{Var}\left(x_{1}\right)+\beta_{2} E\left[\left(\gamma_{1} x_{1}+\gamma_{2} x_{2}+v_{1}\right)\left(x_{1}\right)\right] \\
& =\beta_{1} \operatorname{Var}\left(x_{1}\right)+\beta_{2} E\left[\gamma_{1} x_{1}^{2}+\gamma_{2} x_{2} x_{1}+v_{1} x_{1}\right] \\
& =\beta_{1} \operatorname{Var}\left(x_{1}\right)+\beta_{2} \gamma_{1} \operatorname{Var}\left(x_{1}\right)+\beta_{2} \gamma_{2} E\left[x_{2} x_{1}\right] \\
& =\beta_{1} \operatorname{Var}\left(x_{1}\right)+\beta_{2} \gamma_{1} \operatorname{Var}\left(x_{1}\right)+\beta_{2} \gamma_{2} E\left[\left(\varphi_{1} x_{1}+\delta_{2}\right)\left(x_{1}\right)\right] \\
& =\beta_{1} \operatorname{Var}\left(x_{1}\right)+\beta_{2} \gamma_{1} \operatorname{Var}\left(x_{1}\right)+\beta_{2} \gamma_{2} E\left[\varphi_{1} x_{1}^{2}+\delta_{2} x_{1}\right] \\
& =\beta_{1} \operatorname{Var}\left(x_{1}\right)+\beta_{2} \gamma_{1} \operatorname{Var}\left(x_{1}\right)+\beta_{2} \gamma_{2} \varphi_{1} \operatorname{Var}\left(x_{1}\right) \\
\hat{\beta}_{1} & =\frac{\operatorname{Cov}\left(y_{1}, x_{1}\right)-\beta_{2} \gamma_{1} \operatorname{Var}\left(x_{1}\right)-\beta_{2} \gamma_{2} \varphi_{1} \operatorname{Var}\left(x_{1}\right)}{\operatorname{Var}\left(x_{1}\right)}
\end{aligned}
$$

which we can double-check by substituting in the model estimates (the option 'list' gives the parameter table in the form of a matrix that can easily be accessed and worked with)

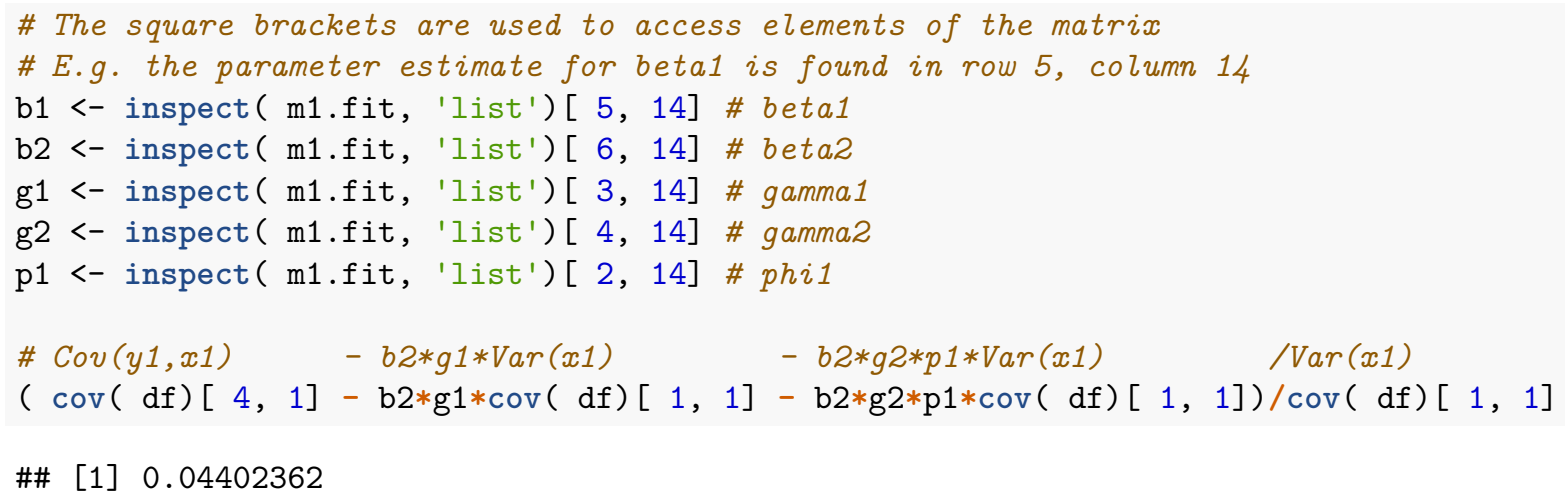

\footnotetext{
${ }^{2}$ Here the covariance algebra is simplified because the variables are all centered with mean zero.
} 
\# Compare with the lavaan estimate of beta1

inspect( m1.fit, 'list') [ 5, 14]

\#\# [1] 0.04402362

The equation for $\hat{\beta}_{1}$ tells us that it is the result of the covariance of $y_{1}$ and $x_{1}$ minus the weighted (by $\hat{\beta}_{2}$ ) covariance of $z_{1}$ and $x_{1}$, divided by the variance of $x_{1}$.

However, as I stated earlier, this procedure is tedious if one wants to inspect the model-implied covariances of each of the elements in the covariance matrix. And the more complex the model in terms of the number of variables and the number of relationships between the variables, the more tedious the procedure becomes.

\section{Ryacas model-implied covariance matrix}

The more efficient option I will present converts the model matrices specified above into the entire symbolic model-implied covariance matrix. This method is efficient because it uses only the matrices $\boldsymbol{\Lambda}, \boldsymbol{B}, \boldsymbol{\Psi}$ and an $(m+n+p+q)^{2}$ identity matrix. The matrices will get larger the more complex the model, but they will not become more numerous.

The first step is to introduce all the model variables (including the errors) and coefficients as symbols using the Sym ( 'variable') function in Ryacas.

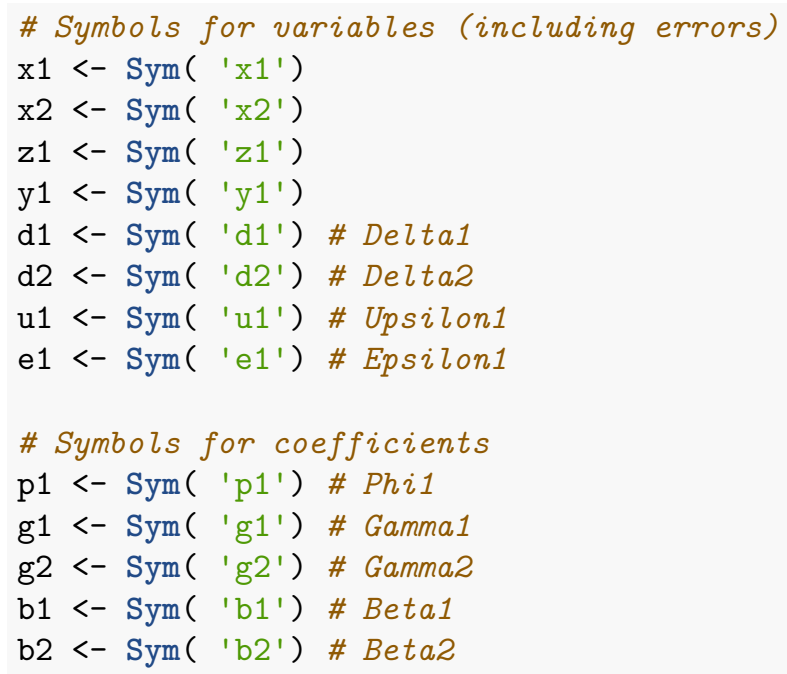

Second, one specifies the model matrices as they were shown above. In Ryacas, vectors are inputted as row vectors using vector $:=\{a, b, c\}$ while for matrices, one uses double curly braces: $\operatorname{matrix}:=\{\{a, b$, $c\},\{d, e, f\}\}$. Matrices are entered row-wise, so element $d$, for example, is found in the second row, first column. Besides defining the symbolic variables, to use Ryacas one must enclose the code in yacas ( " ").

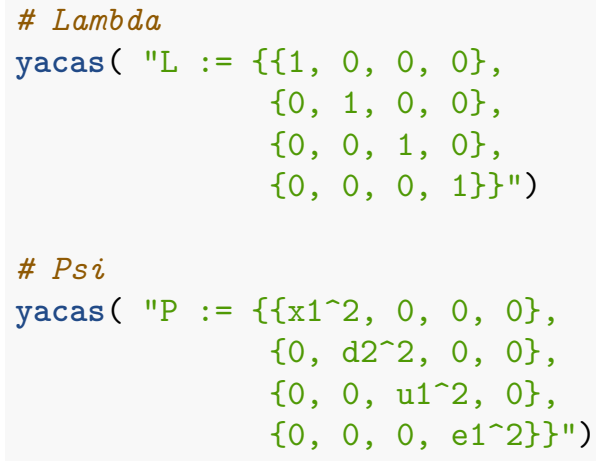




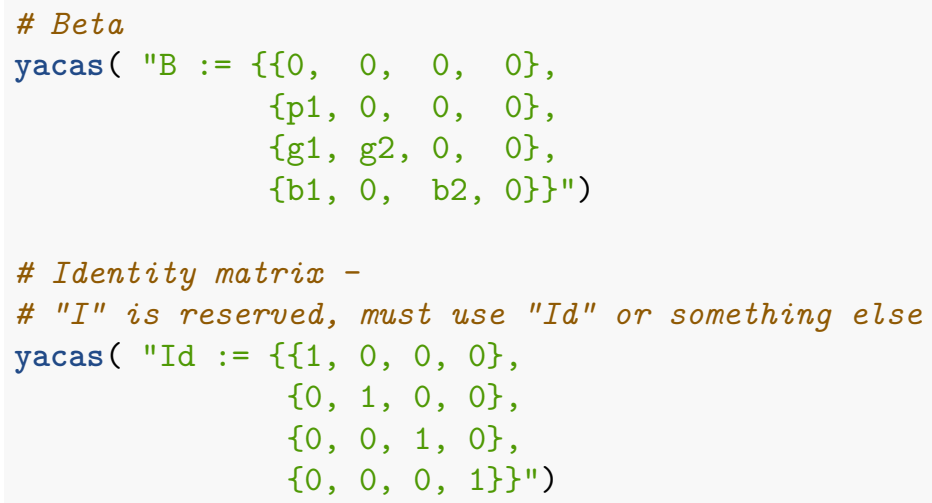

After inputting the model matrices, the equation for $\boldsymbol{\Sigma}(\boldsymbol{\theta})$ can be derived straightforwardly. Note that matrix multiplication works with Dot ( matrix1, matrix2), and that the functions in Ryacas are case sensitive. The transpose and inverse of a matrix works with Transpose ( matrix) and Inverse ( matrix), respectively. Remember, the equation for the model-implied covariance matrix was $\boldsymbol{\Lambda}(\boldsymbol{I}-\mathbf{B})^{-1} \boldsymbol{\Psi}(\boldsymbol{I}-\mathbf{B})^{-1 \prime} \boldsymbol{\Lambda}^{\prime}$

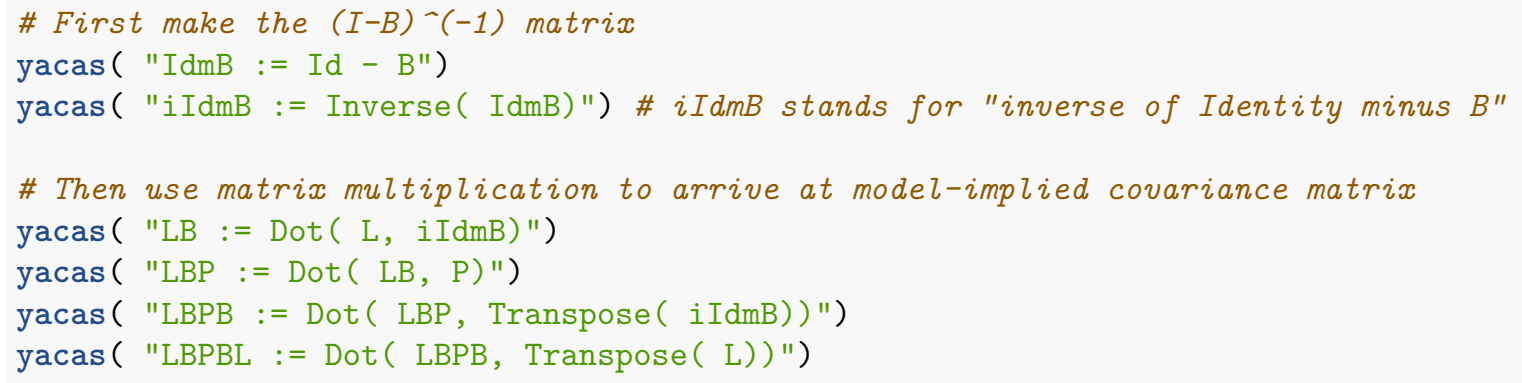

Doing so results in the symbolic model-implied matrix.

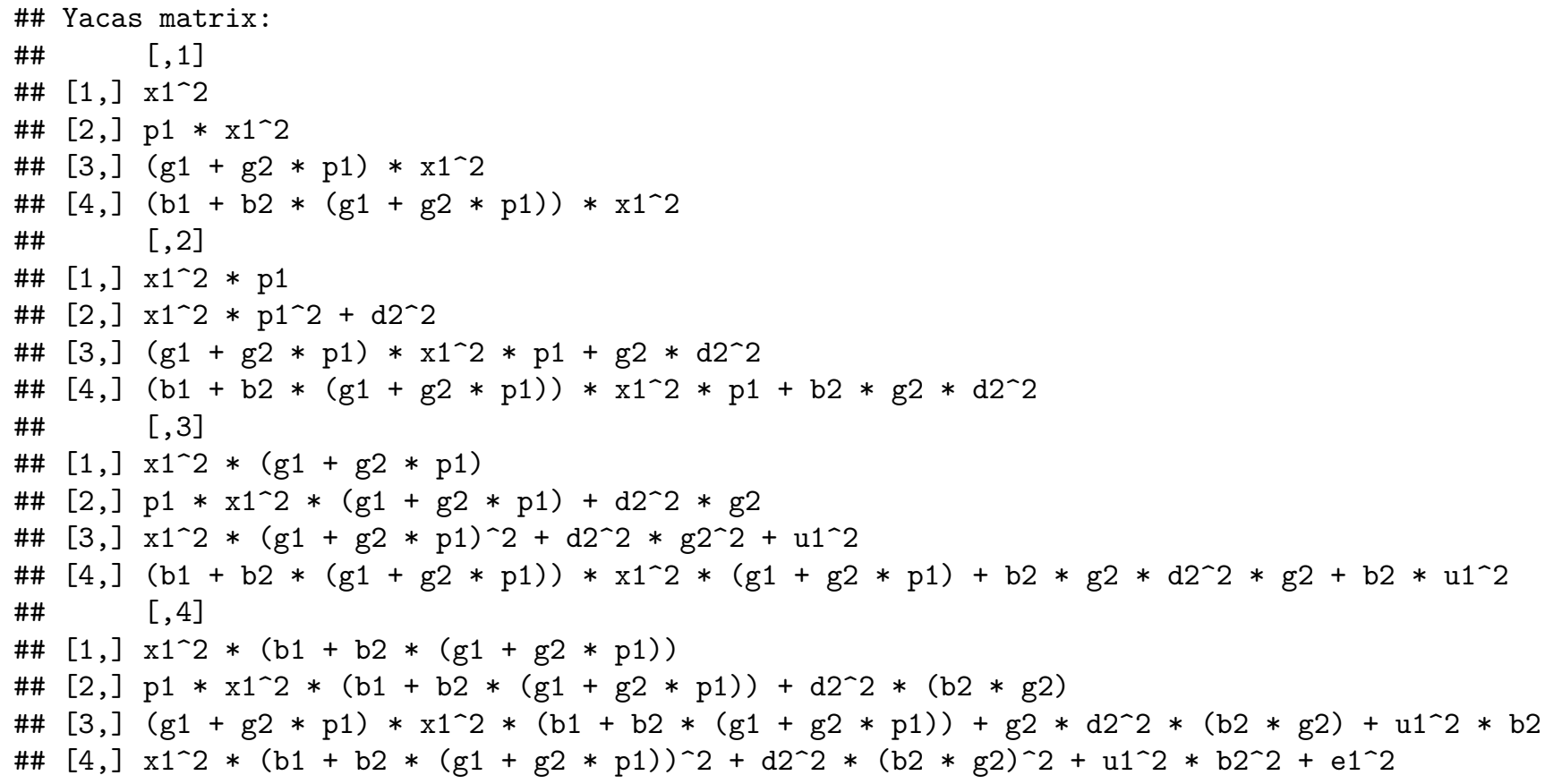

Again, we can double check and see if the proper symbolic model-implied covariance matrix resulted by 


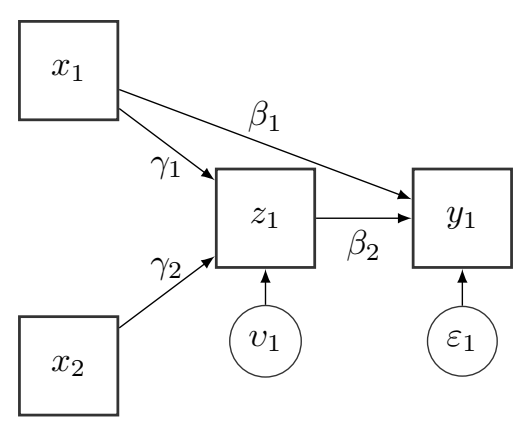

Figure 2: Alternative Model Path diagram

looking, for example, at $\operatorname{Cov}\left(y_{1}, x_{1}\right)$ in the fourth row, first column of the results

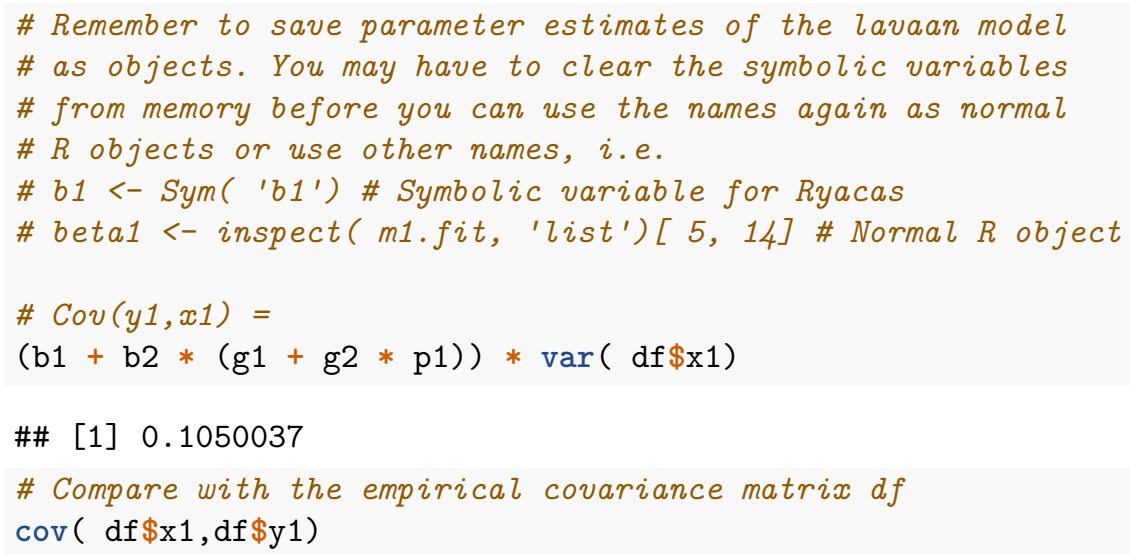

\section{Using the analytical model-implied covariance matrix to gain a better under- standing of SEM models}

Regardless of whether one uses the tracing rule, writes out the covariances algebraically or generates the entire symbolic matrix with the method above, it is important to carefully inspect the model-implied covariance matrix. Doing so can reveal things that are often hidden even in otherwise accurate path diagrams.

For example, suppose we believed the variables $x_{1}$ and $x_{2}$ in the first model were unrelated. Our path diagram would need to be updated to reflect this, see Figure 2.

The question now is, which of the following two scenarios is true?

$$
\begin{aligned}
& z_{1}=\gamma_{1} x_{1}+v_{1_{a}} \\
& z_{1}=\gamma_{2} x_{2}+v_{1_{b}}
\end{aligned}
$$

or

$$
z_{1}=\gamma_{1} x_{1}+\gamma_{2} x_{2}+v_{1}
$$

In other words, are the estimated coefficients $\gamma_{1}$ and $\gamma_{2}$ both total bivariate effects or are they partial coefficients? Could it be that the software is actually basically running two separate bivariate equations for 
$z_{1}$ ? After all, the covariance between $x_{1}$ and $x_{2}$ is shown as zero in the model matrix $\boldsymbol{\Psi} .{ }^{3}$ The answer may be clear to some, but the path diagram does not help us in any way, nor do the typical maximum-likelihood based explanations one often hears along the lines of 'then the optimal estimates are found in an iterative process'.

One way to approach this problem is to inspect the model-implied covariance matrix and check. We can run a second lavaan model, this time without the effect $x_{1} \stackrel{\varphi_{1}}{\longrightarrow} x_{2}$ and again generate the model-implied covariance matrix.

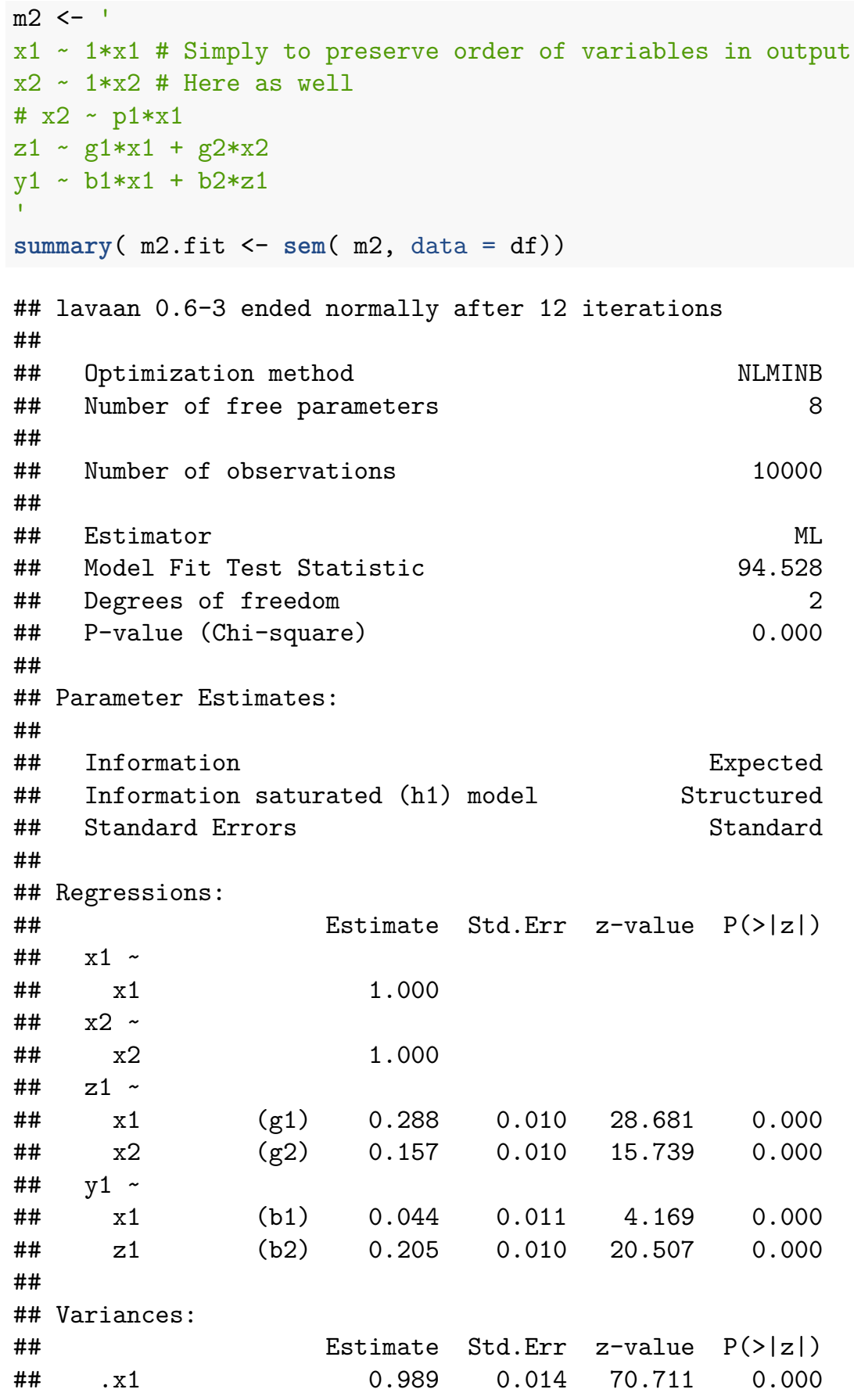

\footnotetext{
${ }^{3}$ This example is motivated by a playful argument between myself and thesis advisor concerning whether to explicitly specify covariances between exogenous predictors.
} 


$\begin{array}{llllll}\# \# & . x 2 & 1.000 & 0.014 & 70.711 & 0.000 \\ \# \# & . z 1 & 0.999 & 0.014 & 70.711 & 0.000 \\ \# \# & . y 1 & 1.021 & 0.014 & 70.711 & 0.000\end{array}$

The model matrices can be viewed again using

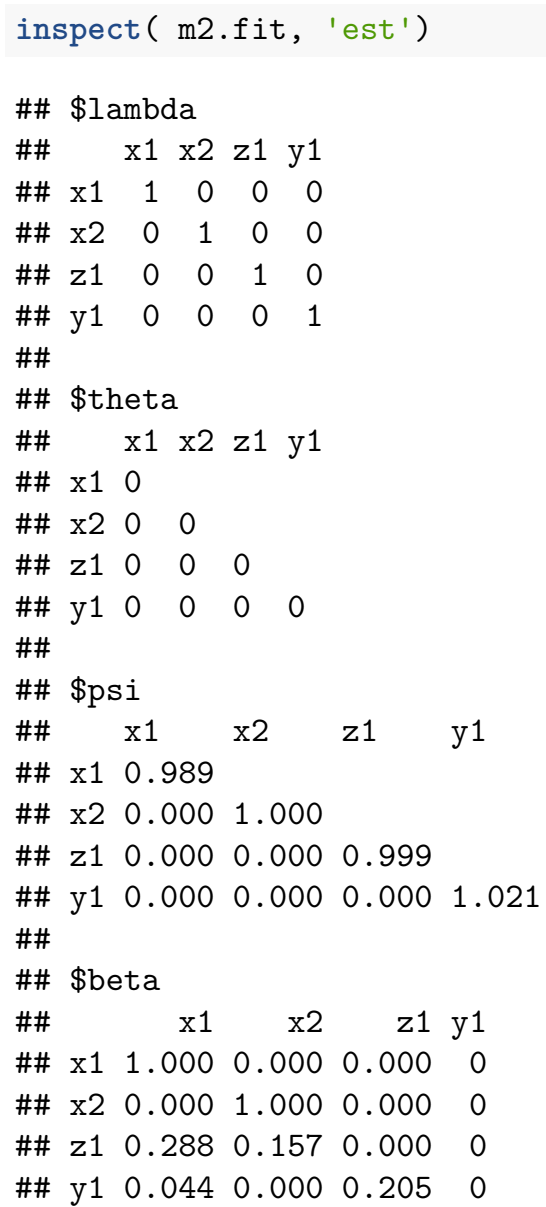

notice the change to the $\boldsymbol{B}$ matrix. Now modify the Ryacas matrix accordingly and generate the symbolic model-implied covariance matrix. All the other matrices stay the same, so as long as they are still in memory one does not need to enter them again.

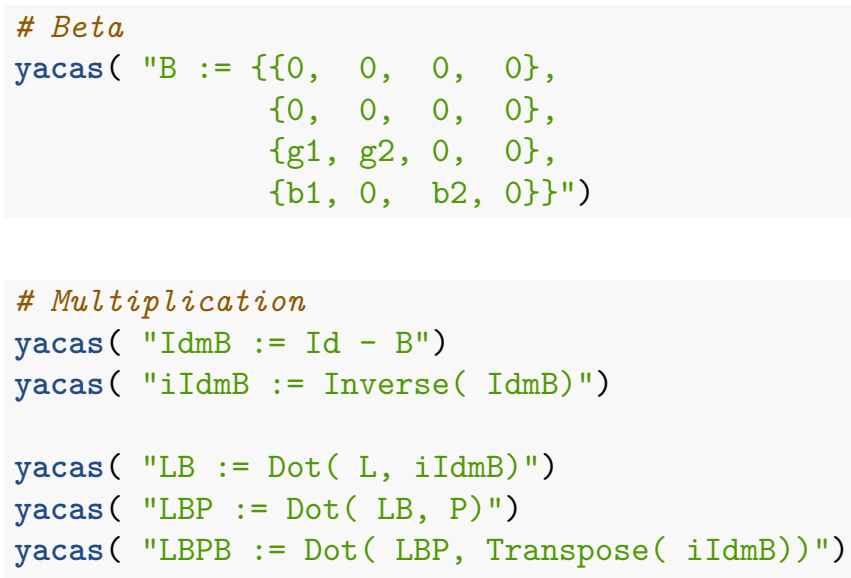




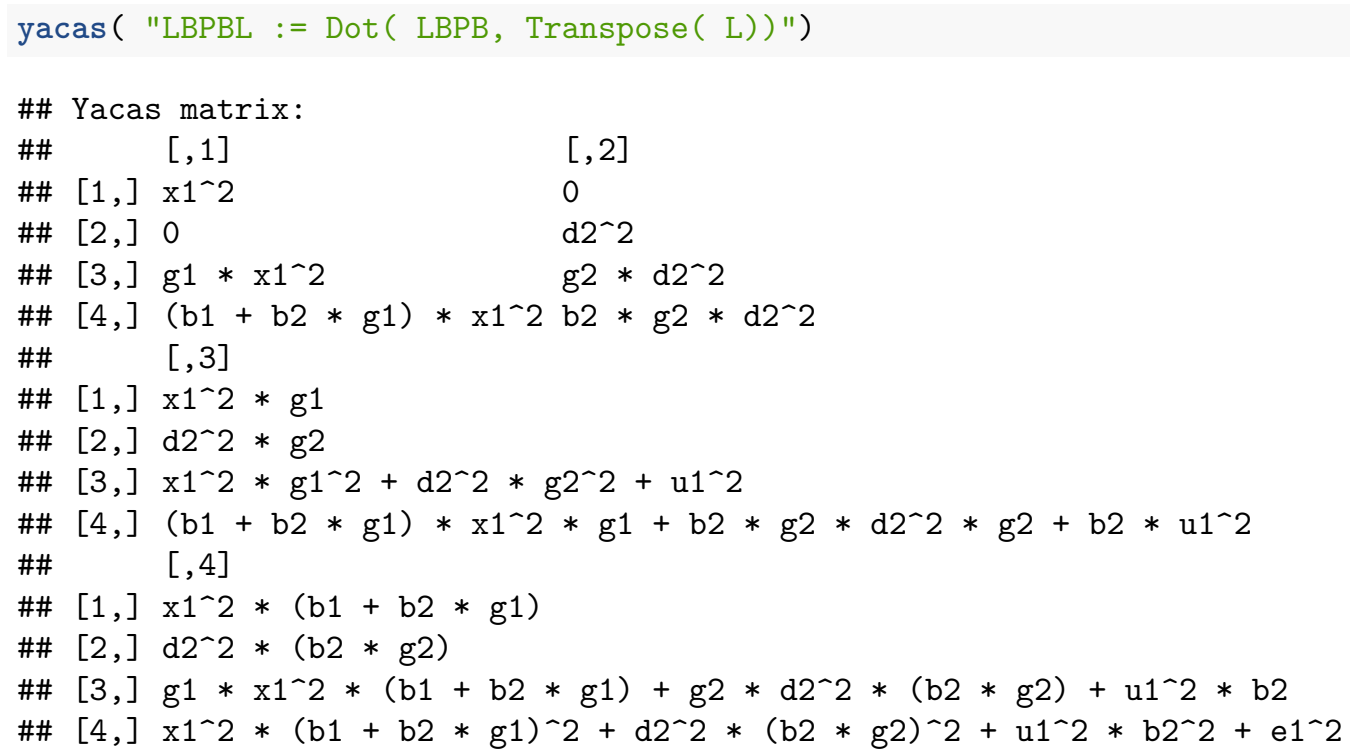

The resulting matrix seems to support the first scenario, that both regression coefficients are uncontrolled bivariate estimates. However, if we substitute in our estimates and solve for $\gamma_{1}$ (as an example, we could do the same for $\gamma_{2}$ ), we see that something is not right.

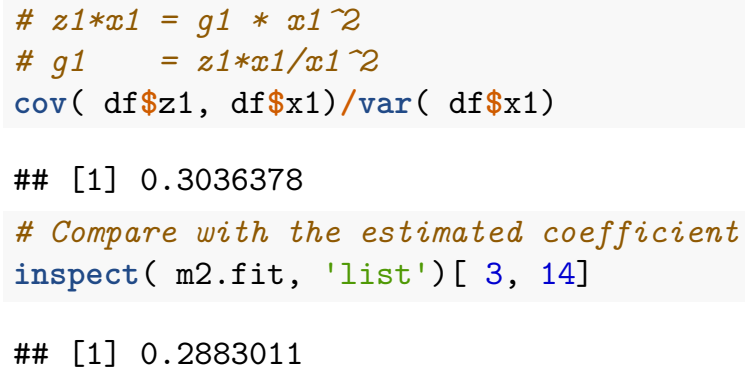

The analytical equation for the coefficient does not equal the estimate from the lavaan model. To check the alternative scenario, that the estimated coefficients are partial coefficients, we can change $\boldsymbol{\Psi}$ to reflect a correlation between $x_{1}$ and $x_{2}$

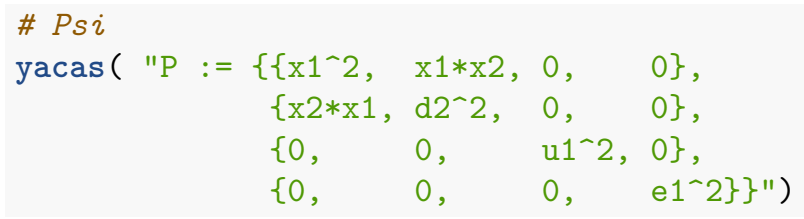

and then re-run the matrix multiplication and see what changes

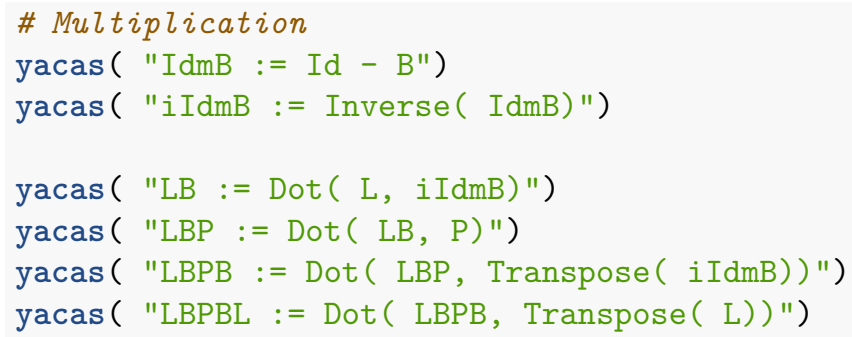




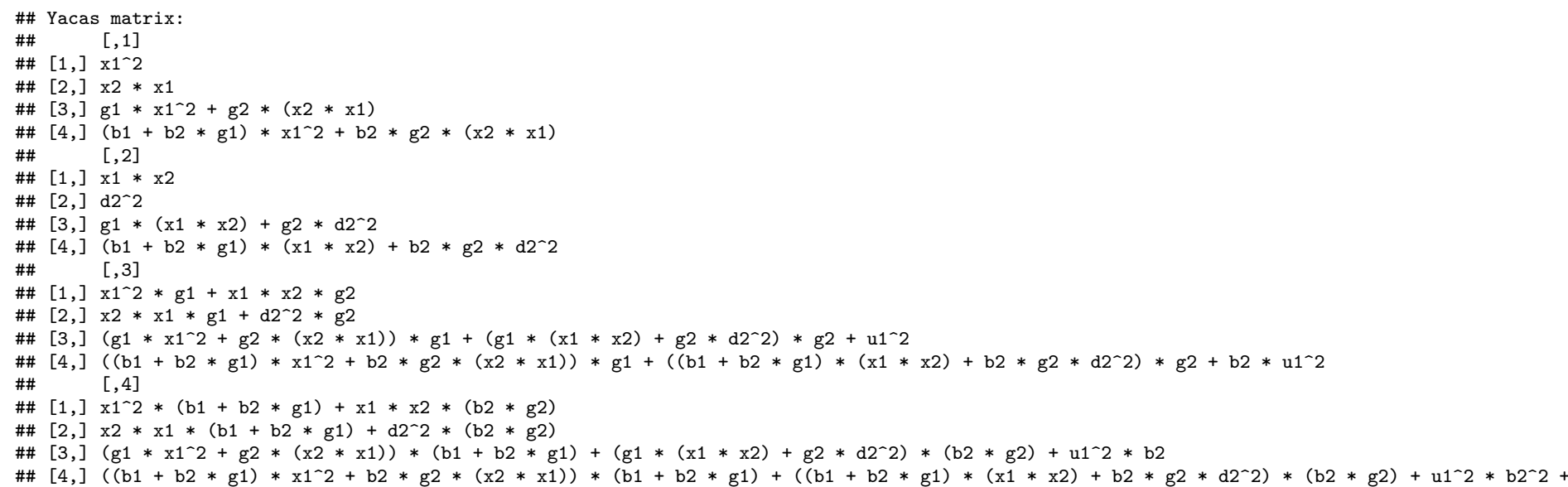

(The equation for $y_{1}^{2}$ gets cut off but that doesn't really matter.)

Now we can solve the alternative equation for $\gamma_{1}$ to show that the estimated coefficients are, in fact, partial coefficients.

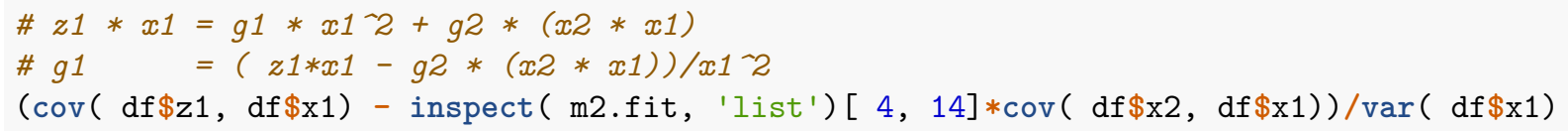

\#\# [1] 0.2883011

From this, we can see that the default behaviour in lavaan is to implicitly account for covariances between exogenous variables with common 'children' to use the language of Judea Pearl (2009). This was not apparent from the path diagram above, nor was it reflected in the lavaan model - even the model matrices showed a 0 in $\boldsymbol{\Psi}$ for the correlation between $x_{1}$ and $x_{2} \cdot{ }^{4}$ However, by taking a closer look at the algebraically derived equation for the parameter of interest and double checking, we were able to conclude that the estimates are in fact partial effects and we learned something about the default behaviour of the software. Upon reflection, it should be clear that this is the only way the model can work but it was not trivial to find conclusive evidence.

\section{Conclusion}

Regardless of the method; tracing, algebraic or using the CAS Ryacas method outlined here, knowing how to generate and inspect the model-implied covariance matrix can be a useful tool. By running the model, specifying the matrices in Ryacas in exactly the same way and deriving the matrix algebra model-implied covariance matrix, we can be sure that no mistakes were made along the way. The tedious algebraic derivation shown above proves that there are many opportunities to make a mistake.

On the other hand, it should be noted that this approaches does not always make sense. For example, the equation for the variance of $y_{1}$ in the last output is probably too long to be of much practical use (although it is accurate!). For a more intutitive understanding of what goes into the variance of that variable, it is likely better to work out the algebra by hand. Then one can decide for themselves when it makes sense to stop expanding. In the algebraic equation for $\operatorname{Cov}\left(y_{1}, x_{1}\right)$ above for example, one could have stopped at line 4 and simply plugged in $\operatorname{Cov}\left(z_{1}, x_{1}\right)$ from the empirical covariance matrix instead of expanding even further. But this is something each researcher must decide for themselves on a case by case basis.

\footnotetext{
${ }^{4}$ It should, however, have been apparent from the fact that the estimates in both of the lavaan models were identical.
} 
The goal of this article was to outline an approach that can compliment the tracing rule and covariance algebra by hand. The benefits are that the CAS approach is efficient, less prone to careless mistakes and that the results are available digitally for further use in $\mathrm{R}$ - results derived by hand need to be entered in to the software manually. In any case, a close inspection of the underlying algebra of the model-implied covariance matrix should be seen as a necessary step in any SEM regression model. It is often difficult to accurately reflect the SEM model in path diagrams alone and there are even situtations where software default settings can be misleading as we have seen from the simple example above.

\section{References}

Andersen, Mikkel, Rob Goedman, Gabor Grothendieck, Søren Højsgaard, Grzegorz Mazur, Ayal Pinkus, and Nemanja Trifunovic. 2019. Ryacas: $R$ Interface to the "Yacas" Computer Algebra System. https: //cran.r-project.org/web/packages/Ryacas/index.html.

Bollen, Kenneth. 1989. Structural Equations with Latent Variables. New York, Chichester: Wiley.

Graff, J. 1979. "Verallgemeinertes LISREL-Modell." Mannheim, Germany.

Kenny, David. 1979. Correlation and Causality. New York: John Wiley \& Sons.

Pearl, Judea. 2009. Causality: Models, Reasoning, and Inference. Second Edition. Cambridge, New York, Melbourne: Cambridge University Press.

R Core Team. 2017. R: A Language and Environment for Statistical Computing. Vienna, Austria: R Foundation for Statistical Computing. https://www.R-project.org/.

Rosseel, Yves. 2012. "lavaan: An R Package for Structural Equation Modeling." Journal of Statistical Software 48 (2): 1-36. http://www.jstatsoft.org/v48/i02/. 